\title{
A Log-Euclidean Polyaffine Registration for Articulated Structures in Medical Images
}

\author{
Miguel Ángel Martín-Fernández, Marcos Martín-Fernández, \\ and Carlos Alberola-López
}

Laboratory of Image Processing, ETSI Telecomunicaciones, University of Valladolid, Campus Miguel Delibes s/n, 47011, Valladolid Spain

\{migmar, marcma, caralb\}@tel.uva.es

\begin{abstract}
In this paper we generalize the Log-Euclidean polyaffine registration framework of Arsigny et al. 1] to deal with articulated structures. This framework has very useful properties as it guarantees the invertibility of smooth geometric transformations. In articulated registration a skeleton model is defined for rigid structures such as bones. The final transformation is affine for the bones and elastic for other tissues in the image. We extend the Arsigny el al.'s method to deal with locally-affine registration of pairs of wires. This enables the possibility of using this registration framework to deal with articulated structures. In this context, the design of the weighting functions, which merge the affine transformations defined for each pair of wires, has a great impact not only on the final result of the registration algorithm, but also on the invertibility of the global elastic transformation. Several experiments, using both synthetic images and hand radiographs, are also presented.
\end{abstract}

\section{Introduction}

Image registration is the determination of a geometric transformation that maps one image into another, aligning objects in both images. Registration within medical imaging is of particular interest [2]. Methods proposed within this field have been classified as frame-based, landmark-based, surface-based, and intensitybased. With respect to the geometrical transformation, methods can be either rigid (only rotations and translations) [3], non-rigid (including scaling, affine transformations, projections and perspective) [4] and elastic transformations [5]. The latter are widely used since local features can be matched while continuity and smoothness in the transformation is maintained.

Very few methods have been proposed in the literature to register images including articulated structures. That is the case for medical images including bones for which rigid transformations are required, but also including surrounding soft tissues for which elastic registration should be used. Papademetris et al. 6] combine several rigid transformations of joints for lower-limb mouse images, obtaining a continuous overall transformation. Baiker et al. 7] propose 
a 3D hierarchical anatomical model in mice skeletal systems. Li et al. 8] propose an algorithm to register whole-body inter-subject volumes for mice and humans. They register the bone structures and then this transformation is refined for the other tissues. du Bois d'Aische et al. [9], present an articulated registration method for the spine column, based on the finite elements method for the elastic deformation outside rigid structures. No invertibility study for these methods, nor an evaluation of the sensitivity of the results with respect to the weighting functions used to fuse the set of rigid transformations are presented.

Arsigny et al. 10] propose a method in which local rigid or affine transformations are combined in a way that the overall transformation is invertible. This is a very important property of the searched transformation. They applied their method to the registration of histological images, arguing that it could also be used for articulated structures. In [1], the authors propose a Log-Euclidean polyaffine transformation approach where its inverse is always guaranteed, being this inverse another Log-Euclidean polyaffine transformation. The authors present a method to merge several affine transformations using normalized weighting functions. Their work focuses on the determination of the global transformation with special stationary properties which provides its invertibility. In [11], the authors proposed Gaussian weights for the regions of influence, where each weight is obtained by means of a mixture of Gaussians placed at some anchor points. In 4], a general framework for articulated registration of images with an inner bonny structure which guarantees exact registration in bone axes while allowing smooth transformation of soft tissues is presented. We propose an iterative process to obtain weights guaranteeing this condition, but no study of the invertibility of overall transformation was performed.

In this paper we generalize the Arsigny et al. 1] Log-Euclidean polyaffine transformation scheme. This scheme guarantees the invertibility of the transformation. However, it cannot be applied directly to articulated registration problems as the affine transformations have to be defined in pairs of landmarks. In the case of articulated registration, each rigid structure is modeled as a wire, and the affine transformation should be applied to pairs of wires. Thus, we propose a generalization of this Log-Euclidean polyaffine transformation to deal with affine wire transformations. This new framework is able to tackle articulated structures such as the bone age assessment problem presented in [4]. We also evaluate the influence of the weighting functions, needed to merge the affine transformations, both in the registration results and in the invertibility of the transformation.

The paper is structured as follows. In section 2 we review articulated registration as well as Log-Euclidean polyaffine registration. We also present novel weighting functions to deal with articulated structures within this polyaffine framework. In section 3 we present several experiments, making a brief discussion about the achieved results. Finally, in section 4 we give some conclusions. 


\section{Method}

\subsection{Articulated Registration}

Let us briefly recall the articulated registration framework, described in [4. The method is a landmark-based elastic registration procedure for anatomical structures that bears an inner skeleton, such as the hand bones. The inner bone skeleton is modeled with a wire model, where wires are drawn by connecting landmarks located in the main joints of the skeletal structure to be registered (long bones). The main feature of this registration method is that it allows to perform affine and elastic transformations on the same image. In this way, in points corresponding to the bone axes (specifically, where the wires are located) an exact registration is guaranteed, while for the remaining image points an elastic registration is carried out.

Fig. 1(a) and Fig. 1(b) show landmarks superimposed on two radiographs. A wire model is built by joining with straight segments (rods) every pair of consecutive landmarks in each finger, following bones axes, as shown in Fig. 1(c).

After obtaining an affine transformation matrix $\mathbf{M}_{\mathbf{i}}$ for each $\operatorname{rod}, R_{i}$, in the model, in order to calculate the final articulated transformation, a weighting of all the transformations is made, with $w_{i}(x, y)$ a measure of how the transformation of $R_{i}$ influences pixel $(x, y)$ in the image. These weights are function of the distance of each image pixel to the rod. This operation can be expressed in homogeneous coordinates as

$$
\left(\begin{array}{lll}
u & v & 1
\end{array}\right)^{T}=\left[\sum_{i=1}^{N} w_{i}(x, y) \mathbf{M}_{\mathbf{i}}\right]\left(\begin{array}{lll}
x & y & 1
\end{array}\right)^{T},
$$

where $(u, v)$ and $(x, y)$ are the Cartesian coordinates of each pixel in the moving image before (input space) and after (output space) the transformation.

To obtain the elastic - articulated - transformation it must satisfy the following conditions [4] (numbered for future reference). (1) the transformation must be affine for pixels nearby the wires, therefore in pixels on $\operatorname{rod} R_{i}$, the weight $w_{i}(x, y)$ corresponding to the $i$-th rod transformation must have a value

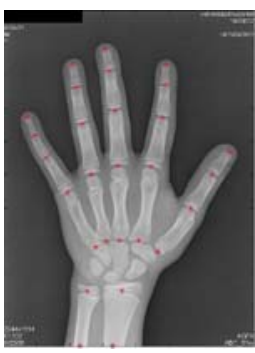

(a)

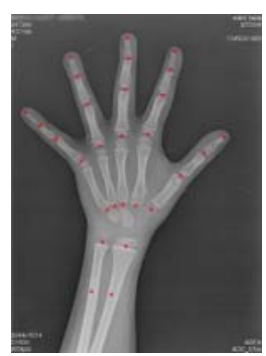

(b)

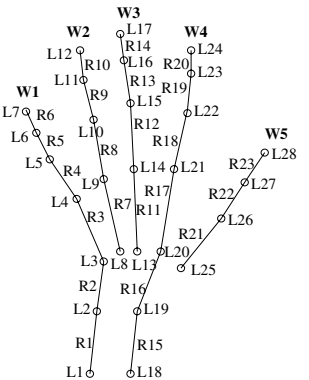

(c)

Fig. 1. (a) Landmark positions superimposed on a radiograph (used as fixed in the experiments); (b) Scheme of the wire model; (c) One example of moving image 
near 1. (2) in pixels on rod $R_{i}$ the other weights, $w_{j}(x, y), j \neq i$, must have a value near 0 . (3) in order to obtain a smooth transformation in points $(x, y)$ far from the rods, the weight $w_{i}(x, y)$ should be defined in inverse proportion with the distance $d_{i}(x, y)$ of that pixel to $\operatorname{rod} R_{i}$. (4) the sum of all the weights in each pixel $(x, y)$ must be unity. To satisfy this last condition, it is enough to normalize, pixelwise, each weight with the sum of all of them.

\subsection{Log-Euclidean Polyaffine Registration}

In 1, the authors extend some previous works 1011 dealing with polyaffine transformation, a mixture of locally affine transformation which fuses to a globally elastic transformation, proposing a Log-Euclidean polyaffine framework for registration. In particular, in [1], they proof that the transformation is invertible, being this inverse a new polyaffine transformation. In addition, they show that the transformation is invariant with respect to coordinate system. This two properties are very desirable in general for the registration of medical images.

The Log-Euclidean polyaffine transformation is proposed as the solution of the ordinary differential equation

$$
\frac{d}{d t}\left(\begin{array}{lll}
x & y & 1
\end{array}\right)^{T}=V(x, y)=\sum_{i=1}^{N} w_{i}(x, y) \log \left(\mathbf{M}_{\mathbf{i}}\right)\left(\begin{array}{lll}
x & y & 1
\end{array}\right)^{T} .
$$

The solution of this equation is always well-defined. The transformed value is given by integrating this equation between 0 and 1 with initial condition the starting point. In case the weights are constant, the solution is $\exp \sum_{i} \log \left(\mathbf{M}_{\mathbf{i}}\right)$, i.e. the Log-Euclidean mean of the components. The solution of this equation has very remarkable properties [1]. The implementation is given by means of the fast polyaffine transform. The speed $V(x)$ is scaled by $2^{N}$ (infinitesimal deformation), then a exponentiation is carried out (approximation) and the solution is given by means of $N$ squarings ( $N$ compositions of the infinitesimal solution). The solution of the infinitesimal equation is approximated by

$$
\mathbf{T}(x, y)=\sum_{i=1}^{N} w_{i}(x, y) \exp \left(\frac{1}{2^{N}} \log \left(\mathbf{M}_{\mathbf{i}}\right)\right)
$$

and then $N$ compositions (squarings) of this transformation is the approximate solution for the Log-Euclidean polyaffine transformation.

\subsection{Weights Definitions}

We propose different weight definitions suited for articulated registration that can be used within the Log-Euclidean framework presented in section 2.2 All the weighting functions depend on the distance $d_{i}(x, y)$ of each pixel $(x, y)$ to each $\operatorname{rod} R_{i}$. We recall to conditions (1)-(4) defined in section 2.1.

Arsigny et al. 11 modeled weights as a mixture of Gaussians located at several anchor points considered as representative of each region. In our case, the representative points of each region are the points on the rod, so we have generalized the Gaussian model to depend on the distance map of each $\operatorname{rod}, d_{i}(x, y)$ 
with mean 0 and standard deviation $\sigma_{i}$, so that weight is maximum in rod pixels, and decays with distance to it. These weights are defined as

$$
w_{i}^{a}(x, y)=G_{\left(0, \sigma_{i}\right)}\left(d_{i}(x, y)\right),
$$

where $G_{(a, \sigma)}$ is a Gaussian function with mean $a$ and standard deviation $\sigma$. These weights satisfy conditions (3) and (4) after normalization, but conditions (1) and (2) are not satisfied as a Gaussian is only zero at an infinite distance.

In 12 we proposed a set of weights. These weights, after an iterative process, satisfy approximately all the conditions (1)-(4). Initial weights $w_{i, 0}^{b}(x, y)$ are calculated as a distance transform, with a value of 1 on the rod and 0 at an infinite distance from it, by means of

$$
w_{i, 0}^{b}(x, y)=\operatorname{sigm}\left(\frac{1}{1+d_{i}(x, y)}\right),
$$

where $\operatorname{sigm}(\cdot)$ is a sigmoid function, which smooths the slope of the weight map near the wire. Finally weights are normalize to satisfy condition (4) using $w_{i, M}^{b}(x, y)$, where $M$ is the total number of iteration steps.

We present here three new weight models that satisfy conditions (1)-(4) by definition, without the need of an iterative process

$$
\begin{gathered}
w_{i}^{c}(x, y)=\exp \left(-\frac{d_{i}(x, y)}{\prod_{j \neq i} d_{j}(x, y)^{\frac{1}{N-1}}}\right), \\
w_{i}^{d}(x, y)=\exp \left(-\frac{d_{i}(x, y)}{\min _{j \neq i} d_{j}(x, y)}\right), \quad w_{i}^{e}(x, y)=G_{(0,1)}\left(\frac{d_{i}(x, y)}{\min _{j \neq i} d_{j}(x, y)}\right),
\end{gathered}
$$

with $N$ the number of rods. These three weights, $w_{i}^{c}, w_{i}^{d}, w_{i}^{e}$, are inversely proportional to $d_{i}(x, y)$ (distance from its rod) and directly proportional to the rest of distances. They have the advantage over $w_{i}^{b}$, that they do not need the iterative process to be, in these cases, exactly 1 on $\operatorname{rod} R_{i}$, and 0 on the other rods, and they do not need any parameter, while $w_{i}^{b}$ and $w_{i}^{a}$ do. Comparing $w_{i}^{c}$ and $w_{i}^{d}$, the first one is proportional to the geometric mean of the rest of distances, while the second one is proportional only to the dominant one, i.e. the minimum one, which has been proven to be more robust. Finally, $w_{i}^{e}$ has lower gradient for pixels near the rod in the same way as sigmoid function in weight $w_{i}^{b}$.

\section{Results and Discussion}

We have applied the articulated registration with the Log-Euclidean polyaffine framework to the synthetic images shown in Fig. 2] using all the weights defined in section 2.3. The weight maps for the horizontal rod, $R_{2}$, are shown in Fig. 3 , It can be observed that $w_{2}^{a}$ does not satisfy conditions (1) and (2), and $w_{2}^{b}$ satisfies them approximately, while the other three ones satisfy them exactly. After normalization, weights $w_{2}^{d}$ and $w_{2}^{e}$ are very similar. 


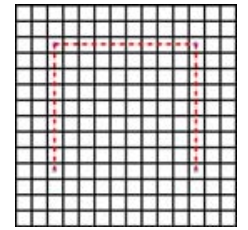

(a) Fixed image.

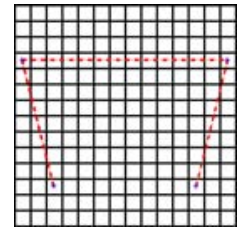

(b) Moving image.

Fig. 2. Synthetic images with wire models superimposed in red and landmarks in blue

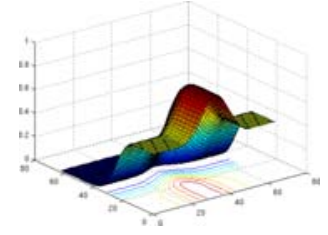

(a) $w_{2}^{a}(x, y)$

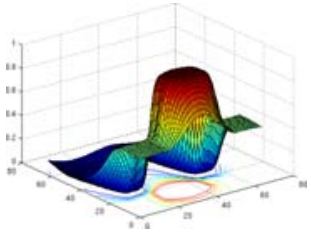

(b) $w_{2}^{b}(x, y)$

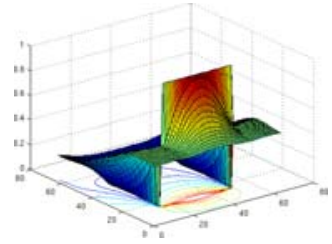

(c) $w_{2}^{c}(x, y)$

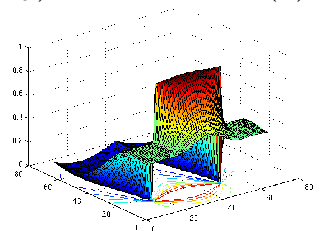

(d) $w_{2}^{d}(x, y)$

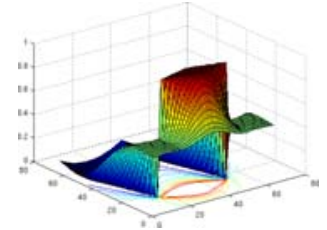

(e) $w_{2}^{e}(x, y)$

Fig. 3. Normalized weight maps corresponding to the horizontal rod $R_{2}$ in Fig. 2(a)
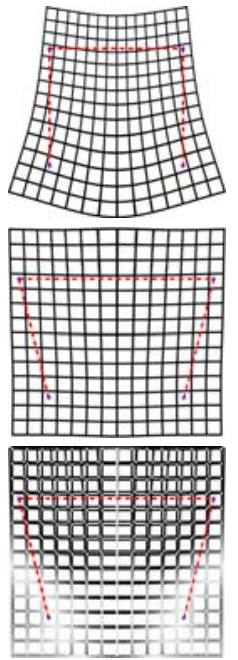

(a) $w_{i}^{a}(x, y)$

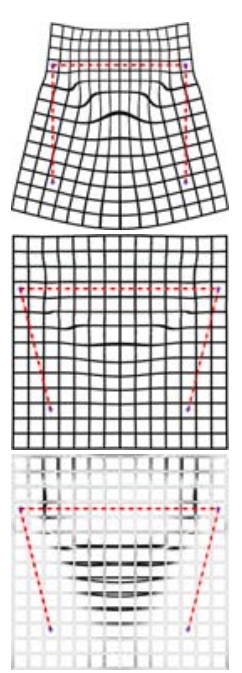

(b) $w_{i}^{b}(x, y)$
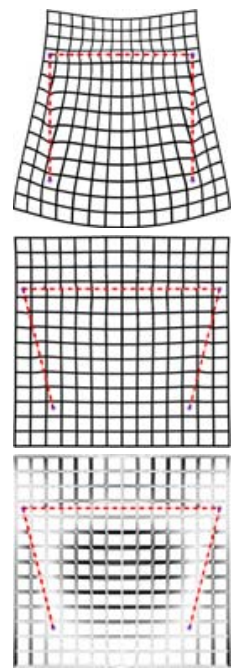

(c) $w_{i}^{c}(x, y)$
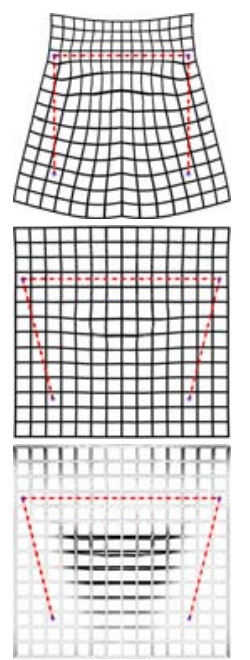

(d) $w_{i}^{d}(x, y)$
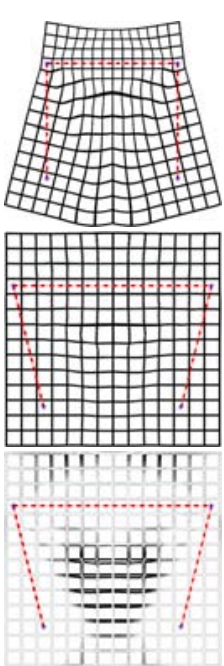

(e) $w_{i}^{e}(x, y)$

Fig. 4. Direct transformation of moving image (upper row), inverse transformation (middle) and difference of inverse transformation with fixed image for images of Fig. 2 
Table 1. Mean and standard deviation of the Mutual Information obtained from 5 radiographs for direct and inverse transformation, when using the different weights

\begin{tabular}{c|c|c|c|c}
\hline & \multicolumn{2}{|c}{ Direct } & \multicolumn{2}{c}{ Inverse } \\
\hline Weight & Mean & Std dev. & Mean & Std dev. \\
\hline$w_{i}^{a}(x, y)$ & 1.32 & 0.11 & 2.65 & 0.32 \\
$w_{i}^{b}(x, y)$ & 1.34 & 0.08 & 2.68 & 0.30 \\
$w_{i}^{c}(x, y)$ & 1.06 & 0.10 & 2.96 & 0.21 \\
$w_{i}^{d}(x, y)$ & 1.47 & 0.07 & 3.27 & 0.38 \\
$w_{i}^{e}(x, y)$ & 1.49 & 0.07 & 3.41 & 0.39 \\
\hline
\end{tabular}

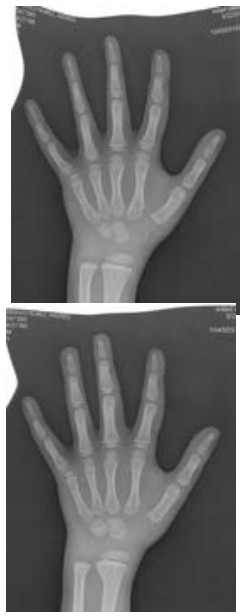

(a) Direct

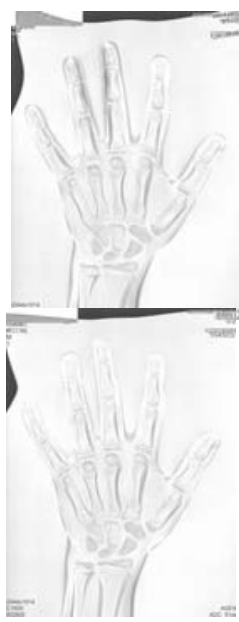

(b) Direct error

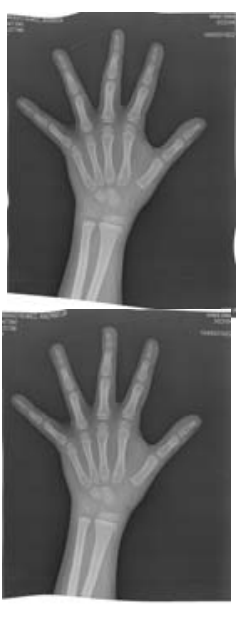

(c) Inverse

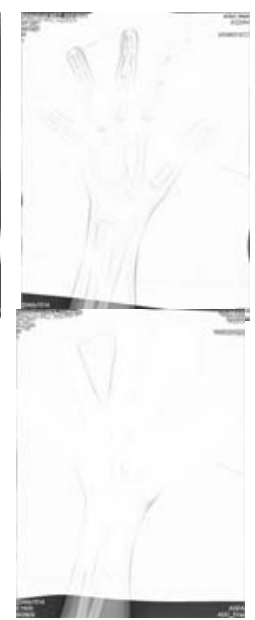

(d) Inverse error

Fig. 5. Graphical registration results for $w_{i}^{a}(x, y)$ (upper row) and $w_{i}^{e}(x, y)$ (lower row)

Transformed and inverse transformed moving image are shown in Fig. 4 together with the difference between moving and inverse transformed image. For the Log-Euclidean polyaffine algorithm $N=6$ squaring steps are employed. It can be seen that $w_{i}^{a}$ does not guarantee exact transformations of pixels near the rods, while all the other ones do. $w_{i}^{b}$ produces the highest deformation in the middle of the rods, causing some residual errors, and $w_{i}^{c}$ produces the most abrupt direct transform. $w_{i}^{d}$ and $w_{i}^{e}$ give similar results.

Finally, we have applied the method to five different hand radiographs which have been registered using image shown in Fig 1(a) as fixed image. $N=6$ squaring steps are also employed. Table 1 shows numerical results of Mutual Information (MI) obtained for direct and inverse registration. Fig. 5 shows the graphical results when Fig 1(c) is used as moving image, with $w_{i}^{a}$ and $w_{i}^{e}$, where it can be seen misregistration produced by $w_{i}^{a}$. With regard to the direct registration, $w_{i}^{e}$ and $w_{i}^{d}$ show the best performance in terms of MI, while $w_{i}^{c}$ shows the worst results. Regarding invertibility of the transformation, $w_{i}^{e}$ shows the best performance, while $w_{i}^{a}$ is the worst one. Graphical results confirm that registration with $w_{i}^{e}$ beats all the others in both directions. 


\section{Conclusions}

We have generalized the Log-Euclidean polyaffine registration framework to articulated structures and compared different weight definitions. From the results obtained we can conclude that Gaussian weights, $w_{i}^{a}$, do not guarantee the rigidity and accuracy of the transformations in the bonny skeleton, while all the others do. Numerical and graphical results confirm that $w_{i}^{e}$ outperforms the other weights in direct and inverse transformations.

Acknowledgments. The authors acknowledge the Spanish CICYT for research grant TEC 2007-67073/TCM. Thanks also go to Dr. Andrés de Llano and Dr. B. Viñuela from Hospital Río Carrión, Palencia, Spain, and Dr. S. Alberola, Centro de Salud Jardinillos, Palencia, Spain, for their valuable comments of the medical aspects of this work, and for the radiographs collected in the Radiology Department of the above mentioned hospital for research purposes.

\section{References}

1. Arsigny, V., Commowick, O., Pennec, X., Ayache, N.: A Log-Euclidean Polyaffine Framework for Locally Rigid or Affine Registration. In: Pluim, J.P.W., Likar, B., Gerritsen, F.A. (eds.) WBIR 2006. LNCS, vol. 4057, pp. 120-127. Springer, Heidelberg (2006)

2. Maintz, J.B.A., Viergever, M.A.: A Survey of Medical Image Registration. Med. Image Anal. 2, 1-36 (1998)

3. Maes, F., Collingnon, A., Vandermeulen, D., Marchal, G., Suetens, P.: Multimodality Image Registration by Maximization of Mutual Information. IEEE Trans. Med. Imaging 16, 187-198 (1997)

4. Martín-Fernández, M.A., Cárdenes, R., Muñoz-Moreno, E., de Luis-García, R., Martín-Fernández, M., Alberola-López, C.: Automatic Articulated Registration of Hand Radiographs. Image Vis. Comput. (2008) doi:10.1016/j.imavis.2008.11.001

5. Rohr, K., Stiehl, H., Buzug, T., Weese, J., Kuhn, M.: Landmark-Based Elastic Registration Using Approximating Thin-Plate Splines. IEEE Trans. Med. Imaging 20, 526-534 (2001)

6. Papademetris, X., Dione, D.P., Dobrucki, L.W., Staib, L.H., Sinusas, A.J.: Articulated Rigid Registration for Serial Lower-Limb Mouse Imaging. In: Duncan, J.S., Gerig, G. (eds.) MICCAI 2005. LNCS, vol. 3750, pp. 919-926. Springer, Heidelberg (2005)

7. Baiker, M., Milles, J., Vossepoel, A., Que, I., Kaijzel, E., Löwik, C., Reiber, J.H.C., Dijkstra, J., Lelieveldt, B.: Fully Automated Whole-Body Registration in Mice Using an Articulated Skeleton Atlas. In: IEEE ISBI, pp. 728-731 (2007)

8. Li, X., Peterson, T.E., Gore, J.C., Dawant, B.M.: Automatic Registration of Whole Body Serial Micro CT Images with a Combination of Point-Based and IntensityBased Registration Techniques. In: IEEE ISBI, pp. 18-25 (2006)

9. du Bois d'Aische, A., Craene, M.D., Geets, X., Gregoire, V., Macq, B., Warfield, S.K.: Estimation of the Deformations Induced by Articulated Bodies: Registration of the Spinal Column. Biomed. Signal Process. and Control 2, 16-24 (2007) 
10. Arsigny, V., Pennec, X., Ayache, N.: Polyrigid and Polyaffine Transformations: A New Class of Diffeomorphisms for Locally Rigid or Affine Registration. In: Ellis, R.E., Peters, T.M. (eds.) MICCAI 2003. LNCS, vol. 2879, pp. 829-837. Springer, Heidelberg (2003)

11. Arsigny, V., Pennec, X., Ayache, N.: Polyrigid and Polyaffine Transformations: a Novel Geometrical Tool to Deal with Non-Rigid Deformations - Application to the Registration of Histological Slices. Med. Image Anal. 9, 507-523 (2005)

12. Martín-Fernández, M.A., Muñoz Moreno, E., Martín-Fernández, M., AlberolaLópez, C.: Articulated Registration: Elastic Registration Based on a Wire-Model. In: Fitzpatrick, J.M., Reinhardt, J.M. (eds.) Medical Imaging 2005: Image Processing. SPIE Press, San Diego (2005) 